\title{
Naturally occurring carcinogens and modulating factors in food of plant origin
}

\author{
W. M. F. Jongen and F. O. Dorgelo ${ }^{1}$ \\ Department of Toxicology, Agricultural University, P.O. Box 8192, 6700 BV Wa- \\ geningen, Netherlands
}

Key words: toxicology, plant breeding, natural carcinogens, modulating factors, mutagenicity, anticarcinogens

\begin{abstract}
In our daily food various carcinogenic and/or mutagenic compounds are present. This conclusion is based on analytical and epidemiological data, experiments in vivo with laboratory animals and mutagenicity assays in vitro. It makes our daily food a major contributing factor in the induction of cancer in humans. According to the widely accepted theory on the origin of cancer, initiating carcinogens (mutagens) and promoters are distinguished. Many natural carcinogens are mutagenic, but not all mutagens are carcinogenic. However, also anticarcinogenic compounds are present in food plants. This leads to a somewhat paradoxal content of our daily food from a carcinogenicity viewpoint. Plant breeding is a possible way to introduce or delete carcinogenic and/or anticarcinogenic principles in food of plant origin.
\end{abstract}

\section{Introduction}

Epidemiological data show that in western societies 1 out of 3 persons gets cancer and 1 out of 4 persons dies of cancer. Doll \& Peto (1981) investigated the factors contributing to the cancer death rate in the United States. About $35 \%$ of this incidence is attributed to nutritional factors. This makes our food a major contributing factor in the induction of cancer. Much information about this relationship is obtained from 'migrant' studies. For example, the population of Japan has a high incidence of stomach cancer and a low incidence of large intestine cancer. An epidemiological study conducted among Japanese immigrants in Hawaii revealed that the pattern of different kinds of cancer is determined mainly by 'environmental' factors (Haenszel et al., 1973). The third generation of this group showed a cancer pattern that was identical to that of the autochthonous people: a low incidence of stomach cancer and a high incidence of large intestine cancer. The term 'environmental' in this context does not mean the pollution grade of the environment by in-

\footnotetext{
${ }^{1}$ Present address: State Institute for Quality Control of Agricultural Products (RIKILT), P.O. Box 230, 6700 AE Wageningen, Netherlands.
} 
dustrial chemicals, but indicates life style factors (e.g. smoking, alcohol) and food habits. This is clearly illustrated in studies conducted among certain religious groups. The seventh-day adventists and the mormones do not consume tea, coffee, alcohol, cigarettes and meat. This life style coincides with a much lower overall cancer death rate.

\section{The process of carcinogenesis}

Generally spoken a compound is called carcinogenic when the use of this compound increases the risk of getting cancer. However it should be emphasized that this does not mean that any factor or compound which, irrespective of the condition, increases the cancer incidence may be called carcinogenic.

There is abundant epidemiological and experimental evidence that the process of carcinogenesis is a multistage process in which two distinct phases can be recognized: the initiation phase and the promotion phase. In the initiation phase an irreversible change in the genetic properties (mutation) of a cell takes place. In the promotion phase the initiated cell acquires a selective growth advantage over the surrounding cells and develops into a visible tumor. Some chemical compounds have both initiating and promoting capacities. These compounds are called complete carcinogens. Other types of compounds, the 'initiators' (mutagens) are able to start the process of carcinogenesis but cannot finish it. On the other hand the 'promoter' compounds cannot start the process but are able to make the initiated cell develop into a visible tumor. The functional differences between an initiator and a promoter arises from their mode of action. Initiators react with DNA thus causing mutations or chromosomal damage. In fact they change the genetic condition of the cells. Promoters do nothing of this kind. They cannot bind to DNA or give rise to mutations. Their mode of action is of a more physiological nature and is membrane-orientated. In fact most promoters influence the differentiation level of the cells and stimulate cell proliferation.

Cancer research of the past decade has shown that a very high percentage of the known carcinogens are also mutagens and that the established assays in vitro for mutagenicity testing have a high predictive value for the carcinogenic potency of a compound. Therefore compounds which have been found mutagenic are suspected of carcinogenic properties.

In the etiology of human cancer not only carcinogens but also modulating factors play an important role. Cocarcinogens (compounds which stimulate the process of carcinogenesis without being carcinogens themselves) and anticarcinogens (compounds which counteract the process of carcinogenesis) are examples of modulating factors.

\section{Naturally occurring carcinogens}

There are a great number of naturally occurring carcinogenic and potentially carcinogenic compounds of plant origin.

In several Asian and African countries the nuts of cycad plants (family Cycada- 


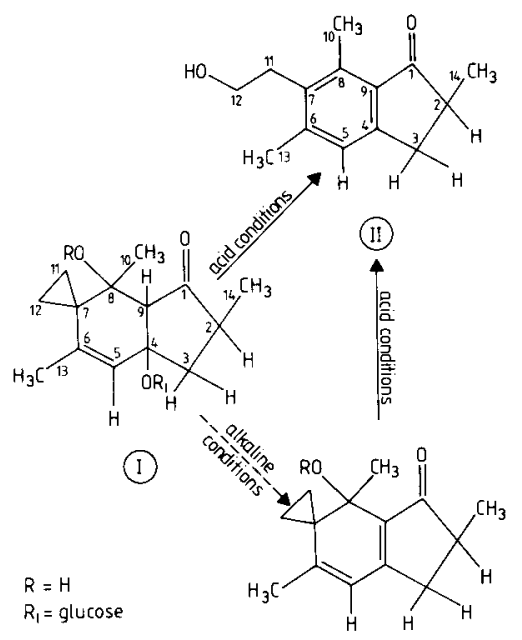

(III)

Fig. 1. Chemical structure of aquilide A (I) and proven (pterosin B, II) or postulated (III) reaction products generated at various conditions as indicated by arrows. Reaction product III is the proposed carcinogenic intermediate formed at $\mathrm{pH}>7$ (van der Hoeven et al., 1983a).

ceae) are used to prepare starch for human consumption. This plant contains cycasin which has been found carcinogenic in various animal species. The amount of cycasin present in cycad nuts depends largely on the species (IARC, 1976).

Bracken fern is a known carcinogenic food plant which is consumed in Japan. It causes intestinal cancer in various animal species and cattle. Epidemiological research in Japan showed that the consumption of this plant is associated with a high incidence of oesophageal cancer. Although intensive research efforts have been made for many years the carcinogenic principle has been identified only recently at the Department of Toxicology of the Agricultural University (van der Hoeven et al., 1983a, c). Almost simultaneously Japanese investigators identified the same compound (Niwa et al., 1983). The structure of the carcinogenic compound is given in Fig. 1.

Carcinogenic pyrrolizidine alkaloids occur in representatives of plants of the families Leguminosae and Compositae. Honey prepared from these plants may contain considerable concentrations of these alkaloids. One quarter of the Australian honey (prepared from Euchium plantagineum) contains up to $1 \mathrm{mg} / \mathrm{kg}$ of the carcinogenic echimidin (Culvenor, 1980; Culvenor et al., 1981). Herbs and spices may contain small amounts of carcinogenic compounds. Safrol occurs in e.g. cacao, black pepper, laurel, nutmeg, mace (IARC, 1976). Safrol has been forbidden as food additive in the USA after animal experiments had revealed that it caused liver tumors in rats.

Allylisothiocyanate (AITC) is the main component in the volatile oil of mustard. AITC occurs also as its thioglycoside sinigrin in plants like cabbage, cauliflower, 
broccoli and Brussels sprouts. AITC has been found carcinogenic for rats (NTP, 1981).

Wild mushrooms contain various hydrazines including gyromitrin. Gyromitrin and related metabolites induce tumors in mice (Toth et al., 1979, 1981). Boiling of the mushrooms destroys more than $99 \%$ of the gyromitrin (Pyysalo, 1975).

\section{Naturally occurring mutagens}

In addition to the carcinogenic plant metabolites mentioned above, food of plant origin contains a number of compounds which are suspected of carcinogenic properties. A large group of such compounds are the flavonoids occurring in all kinds of food plants like lettuce and beans. At this moment there are at least 2000 different flavonoids described (Harborne \& Williams, 1976). The average daily intake in western diets is estimated to be ca. $1 \mathrm{~g}$ (Kuhnau, 1976). Quercetin is a representative of this group which is the most thoroughly investigated. Its daily intake is estimated to be ca. $50 \mathrm{mg}$, and this compound has been found mutagenic in many assays in vitro (Brown, 1980). The data on the carcinogenicity are very contradictory. Pamukcu et al. (1980) reported that quercetin is a potent carcinogen for rats. Exposure to $0.1 \%$ in the diet resulted within 58 weeks in a high incidence of small intestine and bladder tumors. However, in several other studies rats, mice and hamsters were exposed to $0.1 \%-10 \%$ quercetin in the diet, and no increased incidence of any tumor was observed (Hirono et al., 1981; Hosaka \& Hirono, 1981; Morino et al., 1982; Saito et al., 1980). The occurrence of quercetin in food plants depends largely on the cultivar. Mutagenicity studies in our department showed a seven-fold difference in quercetin content in different cultivars. Other plant metabolites which are potentially carcinogenic are found in the group of the anthraquinones and the furocoumarins. Anthraquinones such as emodin occur in rhubarb and senna peas (Mathis, 1966). Emodine and related anthraquinones have been found mutagenic (Brown, 1980). No data are available on the carcinogenic properties of naturally occurring anthraquinones. Furocoumarins or psoralenes occur in parsley, parsnip, celery, dill, fig and fennel; they are also applied in sunprotective oils. Furocoumarins have been found mutagenic in diverse mutagenicity assays (Arlett, 1977; Bridges et al., 1978). In combination with UV light 8-methoxypsoralene has been found carcinogenic for animals and humans (Burger \& Simons, 1978; Stern et al., 1979).

\section{Endogenous formation of mutagens/carcinogens}

In this section we look primarily to the formation of $N$-nitroso compounds in the stomach out of nitrite and amines/amides originating from plant and fish products. The nitrite originates mainly from nitrate that is consumed via vegetables. In the mouth the nitrate is reduced to nitrite by bacteria. In humans the non-reduced nitrate is recirculated and excreted again in the mouth via the salivary gland. In this way a relatively high percentage of the nitrate is reduced to nitrite. A few hours after consumption of purslane, the concentration of nitrite in the saliva from humans 
may increase from $5 \mathrm{mg} / \mathrm{l}$ to $200 \mathrm{mg} / \mathrm{l}$.

In general two different classes of $N$-nitroso compounds can be distinguished. The nitrosamines have to be metabolized before the carcinogenic metabolite is released, while nitrosamides react directly. Exact data on the amounts of $N$-nitroso compounds which are formed in this way are still relatively scarce.

Experiments with simulated gastric juice showed that fish products and also food of plant origin can give rise to the formation of $N$-nitroso compounds. Very recently Nagao (1985) reported that incubation of soya sauce with nitrite resulted in the formation of direct mutagenicity. The responsible precursor compound could be identified as indole-3-carbinol. Epidemiological data indicate that in Colombia the consumption of fava beans is associated with a high incidence of gastric cancer. Incubation of fava beans under simulated gastric conditions in the presence of nitrite resulted in the formation of mutagens (Piacek-Llanes et al., 1982). Only recently, Yang et al. (1985) identified the precursor of the mutagenic compound as a chlorinated indole compound.

\section{Cocarcinogens and promoters}

On the Caribbean island Curacao the black and creole population is burdened with an exceedingly high rate of oesophageal cancer compared with the incidence on surrounding islands. Through epidemiologic investigations it was established that on the island, as part of local lifestyle, the fresh green, aromatic leaves of the bush Croton flavens $\mathrm{L}$. are used to make a 'bush tea' drunk as an everyday beverage. The leaves of this plant contain a large number of tumor promoters including $12-\mathrm{O}$-tetradecanoylphorbol-13-acetate (TPA), the strongest known tumor promoter (Weber \& Hecker, 1978). So far relatively little research has been performed towards the presence of tumor promoters in food of plant origin. It seems reasonable to suppose that in future research a number of unknown promoters will be discovered (Sugimura, 1982a, b).

\section{Anticarcinogens}

Inhibition of the process of carcinogenesis can occur via various mechanisms. The importance of anticarcinogenic compounds may become apparent from the following example. Dutch men have a death rate for lung cancer four times as high as Japanese men. The percentage smokers, which is considered to be the major contributing factor in lung cancer incidence, is equal in both countries. The protective effect is ascribed to a much higher consumption of $\beta$-carotene in Japan (Keys, 1980).

Epidemiological and experimental research has revealed that a number of plant metabolites have a protective effect against the incidence of colon cancer. Especially indole-3-carbinol, diindolylmethane and indole-3-acetonitrile are shown to be active anticarcinogens. At our department, studies in vitro were conducted on the mechanism of this phenomenon. Probably the protective effects of these compounds are due to a change in the balance of enzymes involved in the biotransformation process. This system is responsible for the metabolism of xenobiotics to faci- 
litate excretion (Jongen et al., 1986).

Carcinogens or their reactive intermediates may also be detoxified by a direct interaction with the anticarcinogen. The carcinogenic intermediate of benzo(a)pyrene is detoxified very efficiently by covalent binding to ellagic acid (Wood et al., 1982; Lesca, 1983). Ellagic acid occurs in a number of food products of plant origin such as grapes.

The highly mutagenic and carcinogenic pyrolysis products of tryptophane, which are formed during the frying of meat, are very efficiently detoxified by unidentified factors present in cabbage, apple and pineapple (Kada et al., 1978; Morita et al., 1978).

The formation of $N$-nitroso compounds in the stomach can be inhibited by vita$\min \mathrm{C}$. Vitamin $\mathrm{C}$ reacts directly with the nitrite, and epidemiologic research has shown that a high consumption of vitamin $\mathrm{C}$ is associated with a lower incidence of gastric cancer.

\section{The role of plant breeding}

In this paper we have tried to show that in food of plant origin many naturally occurring carcinogens and modulating factors may exist. In the past few years, research performed at the Department of Toxicology has been focused on the occurrence of varietal differences with respect to the presence of mutagenic principles in food plants. In collaboration with the Institute for Horticultural Plant Breeding, several vegetables commonly consumed in the Netherlands have been investigated. Table 1 gives the results obtained with lettuce and rhubarb. In lettuce, the mutagenic activity which is due to the presence of rutin (the glycoside of quercetin) varies eightfold. In rhubarb, a varietal difference of nine times was observed. A considerable part of the mutagenicity observed in rhubarb could be ascribed to the presence of emodin.

Table 1. Mutagenicity of extracts of 5 cultivars of lettuce and 4 cultivars of rhubarb in the Salmonella/ microsome assay ${ }^{a}$ (van der Hoeven et al., 1983b).

\begin{tabular}{|c|c|c|c|c|}
\hline \multicolumn{2}{|l|}{ Lettuce } & \multicolumn{3}{|l|}{ Rhubarb } \\
\hline cultivar & $\begin{array}{l}\text { revertants } / 150 \mathrm{mg} \\
\text { lettuce }+ \text { GFE }\end{array}$ & cultivar & $\begin{array}{l}\text { revertants } / 132 \mathrm{mg} \\
\text { rhubarb + GFE }\end{array}$ & $\begin{array}{l}\text { revertants } / 200 \mathrm{mg} \\
\text { rhubarb }+\mathrm{S} 9\end{array}$ \\
\hline Sonate & $132^{\mathrm{b}}$ & Paragon & $126^{\mathrm{b}}$ & $120^{\mathrm{b}}$ \\
\hline Ravel & $42^{b}$ & Goliath & 13 & $20^{\mathrm{b}}$ \\
\hline Renate & $346^{\mathrm{b}}$ & Champagne Rood & 27 & $23^{\mathrm{b}}$ \\
\hline Dandy & $169^{\mathrm{b}}$ & Timperley Early & $157^{\mathrm{b}}$ & $176^{\mathrm{b}}$ \\
\hline Deci minor & $98^{\mathrm{b}}$ & & & \\
\hline
\end{tabular}

${ }^{a}$ Used mutant strains of Salmonella typhimurium are not able to synthetize histidine (essential amino acid). Mutagenic compounds can induce reverse mutations in the strains back to the wild type growing without histidine. The number of revertants per plate (visible colonies) is a quantitative measure for the mutagenic potency of the compound under these circumstances. Rat liver enzyme (S9) or gut flora extract (GFE) is added to perform biotransformation of the compounds into active mutagens.

${ }^{b}$ Mutagenic response (over two times the number of revertants in a control experiment). 
Table 2. Mutagenic activity in some cultivars of fava beans after incubation in simulated gastric juice with nitrite ( $\mathrm{pH} 1.5$ ) in the Salmonella/microsome assay (van der Hoeven et al., 1984).

\begin{tabular}{ll}
\hline Cultivar & Revertants/62.5 mg beans \\
White cooking & \\
1 & $1095^{\mathrm{b}}$ \\
2 & $865^{\mathrm{b}}$ \\
14 & $850^{\mathrm{b}}$ \\
3 & $845^{\mathrm{b}}$ \\
& \\
Brown cooking & \\
4 & $370^{\mathrm{b}}$ \\
5 & $415^{\mathrm{b}}$ \\
6 & $380^{\mathrm{b}}$ \\
15 & $440^{\mathrm{b}}$ \\
16 & $375^{\mathrm{b}}$ \\
\hline $\mathrm{a}, \mathrm{b}$ See Table 1. &
\end{tabular}

In a study in collaboration with the Centre for Agrobiological Research also the varietal difference in fava beans was investigated. A more than fifteenfold difference in mutagenic response was observed for various cultivars tested (Table 2). As the data show, good indications have been obtained that varietal differences exist with respect to the presence of mutagenic principles in food products of plant origin. This holds for plants like lettuce, string beans, rhubarb, paprika and fava beans (van der Hoeven et al., 1983b). Because in the studies mentioned the plants were grown under identical conditions and treated with standardized methods the observed differences are due to intrinsic mutagenic properties of the vegetables.

A good example of the influence of plant breeding on the quality of food of plant origin is demonstrated with fava beans. Recently plant breeders have developed white cooking cultivars. These cultivars have been brought on the market and superseded the use of brown cooking cultivars although the latter are considerably less mutagenic after treatment with nitrite.

Differences in intrinsic mutagenicity in food plants may also originate from efforts in plant breeding to obtain more disease-resistant cultivars. In this way new 'chemicals' can be introduced which increase the pre-infectional defence potential of the food plant. Another possibility is that the ability of plants to produce phytoalexins upon fungal contamination or stress may be increased. A preliminary indication that the occurrence of phytoalexins, as a consequence of stress damage, may influence the mutagenic potential of plants is reported for string beans by van der Hoeven et al. (1983b).

This type of research should receive more toxicological attention in the future. On the other hand, plant breeding could play a significant role in cancer prevention by developing cultivars with low intrinsic mutagenic and/or carcinogenic properties. 


\section{Conclusions}

An important aspect with regard to the toxicological impact of plant metabolites is their matrix. For example, cruciferous plants contain sinigrin, which is the glycoside of AITC. In the intestine, the sugar moiety is removed and AITC, which is carcinogenic in animals, is released. On the other hand, also indoles are present which have anticarcinogenic properties. The wholesomeness of a specific food product is difficult to assess from toxicity data of one single compound occurring in this food product.

So far toxicological research on the relation between nutrition and cancer has been concentrated primarily on a systematic screening of mutagenic and/or carcinogenic properties of food products. However, an adequate risk estimation cannot be made without an understanding of the mechanism. Therefore, future research on the Department of Toxicology will focus on mechanistic aspects of the relation between nutrition and cancer. Another point of attention will be the field of tumor promotion. Due to a lack of adequate test systems in vitro, so far no systematic research has been performed towards compounds with promoter activity. Recently promising methods in vitro have been developed which will enable us to get a better insight into the importance of this field with regard to the relation between nutrition and cancer.

\section{Acknowledgement}

We would like to thank Mr W. M. J. van Gelder (Foundation of Agricultural Plant Breeding) for valuable criticism.

\section{References}

Arlett, C. F., 1977. Mutagenicity in cultured mammalian cells. In: D. Scott, B. A. Bridges \& F. H. Sobels (Eds.), Progress in genetic toxicology, p. 141-155. Elsevier/North Holland, Amsterdam.

Bridges, B. A., R. P. Mothershead \& C. F. Arlett, 1978. 8-Methoxypsoralen as a frame-shift mutagen in bacteria and Chinese hamster cells in the dark. Implication for genetic risk in man. Mutation Research 53: 156 .

Brown, J. R., 1980. A review of the genetic effects of naturally occurring flavonoids, anthraquinones and related compounds. Mutation Research 75: 243-277.

Burger, P. M. \& J. W. I. M. Simons, 1978. Mutagenicity and carcinogenicity of 8-MOP/UVA in cell cultures. Bulletin du Cancer 65: 281-282.

Culvenor, C. C. J., 1980. Structure and toxicity of the alkaloids of Russian comfrey (Symphytum erplandicum Nyman), a medicinal herb and item of human diet. Experientia 36: 370-377.

Culvenor, C. C. J., J. A. Edgar \& L. W. Smith, 1981. Pyrrolizidine alkaloids in honey from Echium plantagineum. Journal of Agricultural and Food Chemistry 29: 958-960.

Doll, R. \& R. Peto, 1981. The cause of cancer: quantitative estimates of avoidable risks of cancer in the United States today. Journal of the National Cancer Institute 66: 1191-1308.

Haenszel, W., J. W. Berg, M. Segi, M. Kurihara \& F. B. Locke, 1973. Large bowel cancer in Hawaiian Japanese. Journal of the National Cancer Institute 51: 1765-1779.

Harborne, J. B. \& C. A. Williams, 1975. Flavone and flavonolglycosides. In: J. B. Harborne, T. J. Mabry \& H. Mabry (Eds.), The flavonoids, p. 376-441. Chapman and Hall, London. 
Hirono, I., I. Ueno, S. Hosaka, H. Takanashi, T. Matsushima, T. Sugimura \& S. Natori, 1981. Carcinogenicity examination of quercetin and rutin in ACI rats. Cancer Letters 13: 15-21.

Hoeven, J. C. M. van der, W. J. Lagerweij, M. A. Posthumus, A. van Veldhuizen \& H. A. J. Holterman, 1983a. Aquilide A, a new mutagenic compound isolated from bracken fern (Pteridium aquilinium (L.) Kuhn). Carcinogenesis 4: 1587-1590.

Hoeven, J. C. M. van der, W. J. Lagerweij, I. M. Bruggeman, F. G. Voragen \& J. H. Koeman, 1983b. Mutagenicity of extracts of some vegetables commonly consumed in the Netherlands. Journal of Agricultural and Food Chemistry 31: 1020-1026.

Hoeven, J. C. M. van der, W. J. Lagerweij, W. M. F. Jongen, M. A. Posthumus, A. van Veldhuizen \& H. A. J. Holterman, 1983c. Aquilide A, a new mutagenic compound isolated from bracken fern (Pteridium aquilium(L.) Kuhn). Abstracts book, 13th annual meeting European Environmental Mutagen Society, Montpellier.

Hoeven, J. C. M. van der, W. J. Lagerweij, A. van Gastel, J. Huitink, R. de Dreu \& L. W. van Broekhoven, 1984. Intercultivar difference with respect to mutagenicity of fava beans (Vicia faba L.) after incubation with nitrite. Mutation Research 130: 391-394.

Hosaka, S. \& I. Hirono, 1981. Carcinogenicity test of quercetin by pulmonaryadenoma bioassay in strain A mice. Gann 72: 327-328.

IARC, 1976. IARC monographs on the evaluation of carcinogenic risk of chemicals to man; some naturally occurring substances, Vol. 10. IARC, Lyon.

Jongen, W. M. F., R. J. Topp, H. G. M. Tiedink \& E. J. Brink, 1986. A cocultivation system as a model for in vitro studies of modulating effects of naturally occurring indoles on the genotoxicity of model compounds. Food and Chemical Toxicology (in press).

Kada, T., K. Morita \& T. Inoue, 1978. Anti-mutagenic action of vegetable factor(s) on the mutagenic principle of tryptophan pyrolysate. Mutation Research 53: 351-353.

Keys, A., 1980. Seven countries: A multivariate analysis of death and coronary heart disease. Harvard University Press, Cambridge, Massachusetts/London.

Kühnau, J., 1976. The flavonoids, a class of semi-essential food components: their role in human nutrition. World Review of Nutrition and Dietetics 24: 117-191.

Lesca, P., 1983. Protective effects of ellagic acid and other plant phenols on benzo(a)pyrene induced neoplasia in mice. Carcinogenesis 4: 1651-1653.

Mathis, C., 1966. Comparative biochemistry of hydroxyquinones. In: T. Swain (Ed.), Comparative phytochemistry, p. 245-270. Academic Press, New York.

Morino, K., N. Matsukara, T. Kawachi, H. Ohgaki, T. Sugimura \& I. Hirono, 1982. Carcinogenicity test of quercetin and rutin in golden hamsters by oral administration. Carcinogenesis 3: 93-97.

Morita, K., M. Hara \& T. Kada, 1978. Studies on the natural desmutagens: screening for vegetable and fruit factors active in inactivation of mutagenic pyrolysis products from amino acids. Agricultural and Biological Chemistry 42: 1235-1238.

Nagao, M., 1985. Mutagenic compounds in soya sauces, coffee and herbal teas, meeting abstract. In: Genetic toxicology of the diet, p. 334 (abstract book). Copenhagen, Denmark.

NTP, 1981. NTP technical report on the carcinogenesis bioassay of allylisothiocyanate. National Cancer Institute/National Toxicology Program, Research Triangle Park, North Carolina.

Niwa, H., M. Ojika, K. Wakamatsu, K. Yamada, S. Ohba, Y. Saito, I. Hirono \& K. Matsushita, 1983. Stereochemistry of ptaquiloside, a novel norsesquiterpene glucoside from bracken, Pteridium aquilium var. Latiusculum. Tetrahedron Letters 24: 5371-5372.

Pamukcu, A. M., S. Yalciner, J. F. Hatcher \& G. T. Bryan, 1980. Quercetin, a rat intestinal and bladder carcinogen present in bracken fern. Cancer Research 40: 3468-3472.

Piacek-Llanes, B. G., D. E. G. Struker \& S. R. Tannenbaum, 1982. $N$-nitrosamides of natural origin. In: Proceedings of the 7th International Meeting on analysis and formation of $N$-nitroso compounds. IARC, Lyon.

Pyysalo, H., 1975. Some new toxic compounds in false morels, Gyromitra esculenta. Naturwissenschaften 62: 395.

Saito, D., A. Shirai, T. Matsushima, T. Sugimura \& I. Hirono, 1980. Test of carcinogenicity of quercetin, a widely distributed mutagen in food. Teratogenesis, Carcinogenesis and Mutagenesis 1: 213-221.

Stern, R. S., L. A. Thibodeau, R. A. Kleinerman, J. A. Parrish, T. B. Fitzpatrick and 22 participating 
investigators, 1979. Risk of cutaneous carcinoma in patients treated with oral methoxsalen photochemotherapy for psoriasis. The New England Journal of Medicine 300: 809-813.

Sugimura, T., 1982a. Mutagens in cooked food. In: C. Flek \& A. Hollaender (Eds.), Genetic toxicology, p. 63-79. Plenum Publishing Corporation, New York/London.

Sugimura, T., 1982b. Potent tumor promoters other than phorbol ester and their significance. Gann 73: 499-507.

Toth, B., 1979. Hepatocarcinogenesis by hydrazine mycotoxins of edible mushrooms. Journal of Toxicology and Environmental Health 5: 193-202.

Toth, B., J. W. Smith \& K. D. Patil, 1981. Cancer induction in mice with acetaldehyde methylformylhydrazone of the false morel mushroom. Journal of the National Cancer Instute 67: 881-887.

Weber, J. \& E. Hecker, 1978. Cocarcinogens of the diterpene ester from Croton flavens L. and oesophageal cancer in Curacao. Experientia (Basel) 34: 679-682.

Wood, A. W., M. T. Huang, R. L. Chang, H. L. Newmark, R. E. Lehr, H. Yagi, J. M. Sayer, D. M. Jerina \& A. H. Conney, 1982. Inhibition of the mutagenicity of bay region diol epoxides of polycyclic aromatic hydrocarbons by naturally occurring plants phenols. Exceptional activity of ellagic acid. Proceedings of the National Academic of Science (USA) 79: 5513-5517.

Yang, D., S. R. Tannenbaum, G. Buchi \& G. C. M. Lee, 1984. 4-Chloro-6-methoxy-indole is the precursor of a potent mutagen (4-chloro-6-methoxy-2-hydroxy-1-nitroso-indolin-3-on oxide) that forms during nitrosation of the fava bean (Vicia faba). Carcinogenesis 5: 1224-1228. 